\title{
SHARP LOGARITHMIC SOBOLEV INEQUALITIES ON GRADIENT SOLITONS AND APPLICATIONS
}

\author{
JOSÉ A. CARRILLO AND LEI NI
}

\begin{abstract}
We show that gradient shrinking, expanding or steady Ricci solitons have potentials leading to suitable reference probability measures on the manifold. For shrinking solitons, as well as expanding soltions with nonnegative Ricci curvature, these reference measures satisfy sharp logarithmic Sobolev inequalities with lower bounds characterized by the geometry of the manifold. The geometric invariant appearing in the sharp lower bound is shown to be nonnegative. We also characterize the expanders when such invariant is zero. In the proof various useful volume growth estimates are also established for gradient shrinking and expanding solitons. In particular, we prove that the asymptotic volume ratio of any gradient shrinking soliton with nonnegative Ricci curvature must be zero.
\end{abstract}

\section{INTRODUCTION}

A complete Riemannian manifold $(M, g)$ is called a gradient shrinking soliton (shrinker) if there exists a (smooth) function $f$, such that its Hessian $f_{i j}$ satisfies

$$
R_{i j}+f_{i j}-\frac{1}{2} g_{i j}=0 .
$$

Here $R_{i j}$ denotes the Ricci curvature. As shown in Theorem 4.1 of [CLN], associated to the metric and the potential function $f$, there exists a family of metrics $g(\eta)$, a solution to Ricci flow

$$
\frac{\partial}{\partial \eta} g(\eta)=-2 \operatorname{Ric}(g(\eta))
$$

with the property that $g(0)=g$, the original metric, and a family of diffeomorphisms $\phi(\eta)$, which is generated by the vector field $X=\frac{1}{\tau} \nabla f$, such that $\phi(0)=$ id and $g(\eta)=\tau(\eta) \phi^{*}(\eta) g$ with $\tau(\eta)=1-\eta$, as well as $f(x, \eta)=\phi^{*}(\eta) f(x)$. Namely there exists a self-similar (shrinking) family of metrics which is a solution to the Ricci flow. The metric $g(\eta)$ and $f(\eta)$, sometimes also written as $g^{\tau}$ and $f^{\tau}$, or simply $g$ and $f$ when the meaning is clear, satisfy that

$$
R_{i j}+f_{i j}-\frac{1}{2 \tau} g_{i j}=0 .
$$

We shall denote by $S(x)$ the scalar curvature and by $d \Gamma_{\tau}$ the volume element of $g^{\tau}$.

Gradient shrinking solitons arise as the singularity models of Ricci flow. The more interesting cases are the noncompact ones. Trivial examples include the Euclidean space $\mathbb{R}^{n}$ and the cylinders $\mathbb{S}^{k} \times \mathbb{R}^{n-k}$ for $k \geq 2$. Non-trivial noncompact examples can be found in, for example [FIK]. There is also a more recent construction of solitons with symmetry in [DW]. The main result of this paper is the following theorem, which generalizes the sharp Logarithmic Sobolev Inequality (LSI) of the Euclidean space $\mathbb{R}^{n}[\mathrm{Gr}]$. This was referred as 
Stam-Gross logarithmic Sobolev inequality in [Vi1, Vi2], where one can also find detailed historic accounts and more complete references.

Theorem 1.1. Assume that $(M, g, f)$ is a gradient shrinking soliton, then:

i) The potential $e^{-f}$ is integrable on $M$ and it can be normalized as

$$
\frac{1}{(4 \pi \tau)^{n / 2}} \int_{M} e^{-f} d \Gamma_{\tau}=1
$$

ii) LSI inequality: There exists a geometric invariant $\mu_{s}$, under isometries, which depends only on the value of $f$ and $S$ at the minimum point of $f$ and is independent of the parameter $\tau$, such that

$$
\int_{M}\left\{\tau\left[|\nabla \psi|_{\tau}^{2}+S(\cdot, \tau)\right]+\psi-n\right\} \rho d \Gamma_{\tau} \geq-\mu_{s}
$$

for any $\tau>0$ and any nonnegative compactly supported smooth function $\rho=\frac{e^{-\psi}}{(4 \pi \tau)^{n / 2}}$ with unit integral on $M$. Moreover, the geometric invariant $\mu_{s}$ in the above inequality is sharp.

iii) If $|\mathrm{Rm}| \leq A$ for some $A>0$, then $\mu_{s} \geq 0$.

We refer to Section 2 for notations and the definition of the invariant $\mu_{s}$. Let us observe that the expression in the LSI makes sense wherever $\rho=0$ (hence $\psi=-\infty$ ) since $\rho \psi=0$ there. This can be seen more easily if the integrand is expressed in terms of $\rho$,

$$
\int_{M}\left\{4 \tau|\nabla \sqrt{\rho}|_{\tau}^{2}+\rho S(\cdot, \tau)-\rho \ln \rho-n \rho-\frac{n}{2} \ln (4 \pi \tau) \rho\right\} d \Gamma_{\tau} \geq-\mu_{s} .
$$

Note also that for the Gaussian soliton, namely $(M, g, f)=\left(\mathbb{R}^{n}, g_{\text {can }}, \frac{1}{4}|x|^{2}\right)$, we get $\mu_{s}=0$. The proof of the inequality uses the Bakry-Emery criterion [BE] for LSI's, as obtained from the so-called HWI inequalities derived by Villani and coauthors in different settings [Vi2], see Section 3 and references therein. Here, the main difficulty resides in showing the necessary bounds on the potential to normalize it as in (1.3) and thus, being able to apply these inequalities, which is done in Section 2. We should point out that Perelman has claimed [P, Remark 3.2] that when a LSI holds on a shrinking solitons, the sharp form can be justified using his entropy formula. This mainly applies to compact shrinkers since for the noncompact case, even a weak form of LSI is not known. For the compact shrinkers, our approach supplies a different argument. An immediate consequence of the theorem is the strong non-collapsing of the gradient shrinking solitons. In the case that $M$ has bounded nonnegative Ricci curvature the sharp LSI of Theorem 1.1 implies LSI's for all scales, not necessarily with sharp constants though, hence the non-collapsing at all scales. Let us finally comment that the sharpness in the third statement of our main theorem means that taking the density $\rho$ to be the normalized potential in the first statement, then the minimum $\mu_{s}$ is attained, see Section 4.

In our analysis of the gradient shrinking solitons we also prove the following result.

Corollary 1.2. Any non-flat gradient shrinking soliton with nonnegative Ricci curvature must have zero asymptotic volume ratio.

This is also done in Section 2. This result, in the case of gradient shrinking solitons, generalizes a previous result of Perelman $[\mathrm{P}]$ on ancient solutions with bounded nonnegative curvature operator. The result of Perelman [P, Proposition 11.4] draws the same conclusion 
for any ancient solutions with bounded nonnegative curvature operator. Let us remark that Perelman also assumes the non-collapsing condition, which however is not needed in the proof. Gradient shrinking solitons are special ancient solutions. However our result is more general than that of Perelman for the following reasons: it cannot be derived from Perelman's since we do not assume that the curvature operator is nonnegative nor bounded only Ricci curvature must be nonnegative. On the other hand, the result of Perelman can be derived out of the result above on gradient steady solitons via the asymptotic solitons [P, Proposition 11.2].

Gradient steady/expanding solitons (expanders) arise also in the singularity analysis of Ricci flow [H1]. The technique employed here yields similar sharp geometric inequalities for expanding solitons as well.

Theorem 1.3. Assume that $(M, g, f)$ is a gradient expanding soliton with $\mathrm{Ric} \geq 0$. Then for any nonnegative $\rho(x)=\frac{e^{-\psi(x)}}{(4 \pi)^{n / 2}}$ with $\int_{M} \rho(x) d \Gamma(x)=1$, we have that

$$
\int_{M}\left(|\nabla \psi|^{2}-3 S+\psi-n\right) \rho d \Gamma \geq-\mu_{e} .
$$

Here $\mu_{e}$ is a geometric constant depending only on the value of $f$ and $S$ at the minimum point of $f$. The inequality is sharp for such $\mu_{e}$. Moreover $\mu_{e} \geq 0$ with equality if and only if $(M, g)$ is isometric to $\mathbb{R}^{n}$.

We refer the readers to Section 5 for notations involved in the above theorem. An equivalent expression of the integrand appeared in a recent interesting preprint of Cao and Hamilton $[\mathrm{CH}]$ on pointwise differential estimates of Li-Yau-Hamilton type.

For expanding solitons, we also obtain a volume estimate, which generalizes a recent result of Hamilton [H2], see also [CLN], asserting that the asymptotic volume ratio of gradient expanding solitons with bounded positive Ricci curvature must be positive. The following is one of our statements.

Corollary 1.4. Assume that $(M, g, f)$ is a gradient expanding soliton with $S(x) \geq-\beta$ for some constant $\beta \geq 0$. Then for any $o \in M$ and $r \geq r_{0}$

$$
V(o, r) \geq V\left(o, r_{0}\right)\left(\frac{r+a}{r_{0}+a}\right)^{n-2 \beta}
$$

with $a=2 \sqrt{f(o)+\mu_{e}+\beta}$.

The above mentioned Hamilton's result follows from the above statement applying to the case $\beta=0$. For general $\beta$, the growth rate in our estimate is sharp as shown by examples. This is proved in Section 5. A similar/independent result can also be found in a recent preprint $[\mathrm{ChT}]$.

For gradient steady soliton, since one can not expect that the LSI holds in general in viewing of Hamilton's 'cigar', we obtain a sharp weighted Poincaré inequality instead. The proof is relatively easy, without appealing the above mentioned theory involving the BakryEmery criterion, and is done in Section 6.

The part $\mu_{s}, \mu_{e} \geq 0$ of the main theorems is finally proved in Section 7. This is motivated by the Zamolodchikov's c-theorem of the re-normalization group flow $[\mathrm{Z}]$. In view of the entropy monotonicity formula of Perelman, its connection with the LSI, as well as the fact that gradient shrinking solitons arise as the singularity models (at least for the cases that 
the blow-up has nonnegative curvature operator), this result can be viewed as an analogue of Zamolodchikov's c-theorem for the re-normalization group flow. The proof makes use a Li-Yau-Hamilton type inequality of Perelman $[\mathrm{P}]$ and the entropy formula/monotonicity for the linear heat equation of [N2].

By the work of Dolbeault and Del Pino [DoPi] (also Toscani and the first author [CT]) the sharp form of Sobolev-Gagliardo-Nirenberg inequalities on $\mathbb{R}^{n}$ are related to the nonlinear Fokker-Planck dynamics of porous medium/fast diffusion type equations. It is interesting to find out if such relation can lead to sharp inequalities on solitons along a similar line of argument of this paper.

\section{Geometric estimates on Gradient Shrinking SOlitons}

We shall follow the notations of the introduction being our first objective to show the integrability on the potential function for solitons allowing for the normalization (1.3). The following equations are simple consequences of the soliton equation (1.2):

$$
\begin{aligned}
S+\Delta f-\frac{n}{2 \tau} & =0, \\
S+|\nabla f|^{2}-\frac{f}{\tau} & =\frac{\mu_{s}(\tau)}{\tau} .
\end{aligned}
$$

where $\mu_{s}(\tau)$ is a constant that will be eventually chosen by the normalization of the potential as in (1.3). Here $S$ is the scalar curvature. See, for example [H1] or [CLN], for a proof. The equations (2.1) and (2.2) imply that

$$
2 \Delta f-|\nabla f|^{2}+S+\frac{f-n}{\tau}=-\frac{\mu_{s}(\tau)}{\tau} .
$$

The lemma below implies that the integral involved in the normalization (1.3), as well as other integrals involved later in the proof of Theorem 1.1, are finite.

Lemma 2.1. Let $r(x)$ be the distance function to a fixed point $o \in M$ with respect to $g(\eta)$ metric. Then there exist constant $C_{1}$ and $C_{2}$ such that

$$
f(x) \geq \frac{1}{4 \tau}\left(r(x)-C_{1}\right)^{2}
$$

for $r(x) \geq C_{2}$ and

$$
f(x) \leq \frac{1}{4 \tau}\left(r(x)+C_{1}\right)^{2}, \quad|\nabla f|(x) \leq \frac{1}{2 \tau}\left(r(x)+C_{1}\right)
$$

for $r(x) \geq C_{2}$.

Proof. First we observe that $S \geq 0$ by a gradient estimate argument of Chen [Ch] (see also the appendix of $[\mathrm{Yo}])$. The estimate (2.4) then follows verbatim from [FMZ, pages 655-656].

Now (2.2) and $S \geq 0$ imply that

$$
|\nabla f| \leq \sqrt{\frac{f+\mu_{s}(\tau)}{\tau}} .
$$

The first estimate in (2.5) follows easily from this by integrating $f+\mu_{s}(\tau)$ along minimizing geodesics from $o$, see also the proof of Proposition 5.1. The second estimate in (2.5) follows from the first one via (2.6). 
Corollary 2.1. Let $(M, g, f)$ be a gradient shrinking soltion. Then the minimum of $f$ can be achieved somewhere, say $o \in M$. Hence $f(o)$ and $S(o)$ are fixed for different $\eta$ and the constant $\mu_{s}(\tau)$ in (2.2) is a constant independent of $\tau$. Therefore $\mu_{s} \doteqdot \mu_{s}(\tau)$ is an invariant of the soliton. Moreover,

$$
\int_{M}\left(|\Delta f|+|\nabla f|^{2}+|f|+S\right) e^{-f} d \Gamma_{\tau}<\infty
$$

Proof. The first part of the corollary is evident since $o$ is the fixed point of $\phi(\eta)$. The change of $S$ (from the shrinking) is compensated by the factor $\tau$. For the second part, observe that by [WW, page 7], we have that

$$
e^{-f} J(r, \theta) \leq A_{1} e^{a_{2} r-\frac{1}{2 \tau} r^{2}}
$$

for some positive constants $A_{1}, a_{2}$ independent of $r$. Here $J(r, \theta)$ is the area element of the sphere $\partial B_{o}(r)$. Namely, Area $\left(\partial B_{o}(r)\right)=\int_{\mathbb{S}^{n-1}} J(r, \theta) d \theta$. Notice that (2.1) and (2.2), along with (2.4) and (2.5) effectively imply that

$$
\begin{aligned}
0 \leq S & \leq \frac{1}{4 \tau^{2}}\left(r(x)+C_{1}\right)^{2}, \\
-\frac{n}{2} \leq-\Delta f & \leq \frac{1}{4 \tau^{2}}\left(r(x)+C_{1}\right)^{2} .
\end{aligned}
$$

Since $\int_{M}(\cdot) d \Gamma_{\tau}=\int_{0}^{\infty} \int_{\mathbb{S}^{n-1}}(\cdot) J(r, \theta) d \theta d r$, the finiteness of the integral in (2.7) follows easily.

Note that by Theorem 4.1 of [CLN], $\frac{e^{-f}}{(4 \pi \tau)^{n / 2}}$ satisfies the conjugate heat equation

$$
\left(\frac{\partial}{\partial \tau}-\Delta+S\right)\left(\frac{e^{-f}}{(4 \pi \tau)^{n / 2}}\right)=0
$$

Hence the total mass of $e^{-f}$, namely the normalization (1.3) is preserved along the evolution. In other words, if

$$
\int_{M} \frac{e^{-f}}{(4 \pi \tau)^{\frac{n}{2}}} d \Gamma_{\tau}=1
$$

holds at $\tau=1$ (which corresponds to $\eta=0$ ), it holds for all $\tau>0$. Also note that $\mu_{s}(M, g)=\mu_{s}\left(M^{\prime}, g^{\prime}\right)$ if $(M, g)$ is isometric to $\left(M^{\prime}, g^{\prime}\right)$ by the virtue of [Na, Lemma 1.2]. Equivalently, the invariant $\mu_{s}(M, g)$ is independent of the choice of the potential function $f$ since the difference of two potential functions is either a constant or a linear function, since they have the same Hessian. In the first case, the normalization make the constant zero. For the second case, namely the difference of the two potential functions is a linear function, then the manifold $M$ splits off a line. Some simple calculation also show that the normalization would make the constants $\mu_{s}$ in (2.2) identical for the two different potential functions. In fact, if the difference of two potential functions $h \doteqdot f_{1}-f_{2}$ (assuming $\tau=1$ without the loss of the generality), is a linear function of $\mathbb{R}$ and $M=\mathbb{R} \times M_{1}$, using the soliton equation (2.1) one can write $f_{k}(x, y)=\frac{1}{4} x^{2}+b_{k} x+c_{k}(y)$ for $k=1,2$. Here we denote the coordinate of $\mathbb{R}$ by $x$ and the coordinate of $M^{\prime}$ by $y$. Since $h(x)=a x+b$ for constant $a$ and $b$. Hence we have $c_{1}(y)-c_{2}(y)=c$. Now if $\int_{M} e^{-f_{1}}=\int_{M} e^{-f_{2}}$, by simple direct calculation we have that $b_{1}^{2}=c+b_{2}^{2}$. Direct calculation shows that

$$
\left(\left|\nabla f_{1}\right|^{2}+S-f_{1}\right)-\left(\left|\nabla f_{2}\right|^{2}+S-f_{2}\right)=b_{1}^{2}-b_{2}^{2}-\left(c_{1}(y)-c_{2}(y)\right)=0 .
$$

The next result concerns the behavior of the volume $V(o, r)$ of balls $B(o, r)$, especially as $r \rightarrow \infty$. We start with the easier case of the Ricci curvature being bounded. 
Corollary 2.2. Let $(M, g)$ be a nonflat gradient shrinking soliton with Ric $\geq 0$. Assume further that the scalar curvature $S(x) \leq C_{1}$ for some $C_{1}>0$. Then, there exists a constant $\delta=\delta(M, f)>0$ with the property that for any $o \in M$, there exists $a=a\left(M, f, C_{1}\right)$ such that for any $r \geq r_{0} \geq a$

$$
V(o, r) \leq V\left(o, r_{0}\right)\left(\frac{r-a}{r_{0}-a}\right)^{n-\delta}
$$

Proof. Without the loss of generality we may assume that $\tau=1$. By [N3, Proposition 1.1], we have $\delta=\delta(M, f)>0$ such that $S \geq \frac{\delta}{2}$. On the other hand, by [P, Section 8], see also the proof of [N3, Proposition 1.1], for any minimizing geodesic joining $o$ to $x=\gamma\left(s_{0}\right)$ with $s_{0} \geq 2$ and $r_{0}>0$ such that $s_{0}-r_{0} \geq 1$, we have that

$$
\int_{0}^{s_{0}-r_{0}} \operatorname{Ric}\left(\gamma^{\prime}, \gamma^{\prime}\right) d s \leq C_{4}(M)+\frac{n-1}{r_{0}} .
$$

This implies, again by the argument in the proof of [N3, Proposition 1], that

$$
\frac{\partial f}{\partial r}(x) \geq \frac{r(x)}{2}-C_{6}(M, f, o) .
$$

Now integration by parts on equation (2.1) over $B(o, r)$ yields that

$$
\begin{aligned}
\frac{n-\delta}{2} V(o, r) & \geq \int_{B(o, r)}\left(\frac{n}{2}-S\right) d \Gamma \\
& =\int_{\partial B(o, r)} \frac{\partial f}{\partial r} d A \\
& \geq A(o, r)\left(\frac{r}{2}-C_{6}\right) .
\end{aligned}
$$

Here $A(o, r)$ is the surface area of $\partial B(o, r)$. The result follows from integrating the above estimate on $\left[r_{0}, r\right]$.

Remark 2.3. Being Corollary 2.2 proved under no restriction on the boundedness of the Ricci curvature, it might be used to prove [P, Proposition 11.4]. This result concludes that any nonflat ancient solution of Ricci flow with bounded nonnegative curvature operator must have the asymptotic volume ratio $\lim _{r \rightarrow \infty} \frac{V(o, r)}{r^{n}}=0$. In fact one can derive $[\mathrm{P}$, Proposition 11.4] by contradiction: Assume the claim is false, one obtains an asymptotic soliton by $[\mathrm{P}$, Proposition 11.2], which is nonflat and that has the maximum volume growth. On one hand, we now may use the volume comparison theorem, to get that $V(o, r) / r^{n}$ is always bounded above the corresponding ratio of the Euclidean space. On the other hand, it is easy to show that the asymptotic volume ratio for an ancient solution with bounded nonnegative curvature is monotone non-increasing in $t$. Hence the asymptotic soliton must has positive asymptotic volume ratio. This is a contradiction with the estimate (2.10).

With some extra effort, we can indeed prove such desired volume estimate without assuming the Ricci curvature upper bound.

Proposition 2.1. Let $(M, g, f)$ be a nonflat gradient shrinking soliton with $\mathrm{Ric} \geq 0$. Then

$$
\lim _{r \rightarrow \infty} \frac{V(o, r)}{r^{n}}=0 .
$$

Here $V(o, r)$ is the volume of $B(o, r)$. 
Proof. We can reduce ourselves to the case $\tau=1$ without loss of generality. For simplicity, after translation we may assume that the potential function $f$ satisfies $|\nabla f|^{2}+S \leq f$. It is also more convenient to work with sub-level sets of $f$. Let us consider the sets

$$
F_{r} \doteqdot\{x \in M \mid 2 \sqrt{f(x)} \leq r\}
$$

and $\widetilde{V}(r)=\operatorname{Vol}\left(F_{r}\right)$. Assume that the conclusion is not true, then $\liminf _{r \rightarrow \infty} r^{-n} V(o, r) \geq$ $\eta>0$ for some $\eta>0$. This clearly implies that $\liminf _{r \rightarrow \infty} r^{-n} \widetilde{V}(r) \geq \eta^{\prime}>0$.

On the other hand, following [CZh, Mu] we consider the function

$$
\chi(r)=\int_{F_{r}} S d \Gamma .
$$

Using $S \geq \delta>0$ for some $\delta>0$, which is ensured by [N3, Proposition 1.1], we have that for any small $\epsilon>0$

$$
\begin{aligned}
\left(n-\delta^{\prime}\right) \widetilde{V}(r)+2(1-\epsilon) \chi(r) & =\int_{F_{r}} 2\left(\frac{n}{2}-\epsilon \delta+(1-\epsilon) S\right) d \Gamma \\
& \geq \int_{F_{r}} 2\left(\frac{n}{2}-S\right) d \Gamma \\
& =\int_{F_{r}} 2 \Delta f d \Gamma \\
& =r \widetilde{V}^{\prime}(r)-\frac{4 \chi^{\prime}(r)}{r} .
\end{aligned}
$$

Here $\delta^{\prime}=2 \epsilon \delta$ and in the last line we used the computation in (4) of [Mu]. Integrating the above estimate as in $[\mathrm{Mu}, \mathrm{CZh}]$, we arrive at

$$
\frac{\widetilde{V}(r)}{r^{n-\delta^{\prime}}}-\frac{\widetilde{V}\left(r_{0}\right)}{r_{0}^{n-\delta^{\prime}}} \leq \frac{4 \chi(r)}{r^{n-\delta^{\prime}+2}}
$$

for $r \geq r_{0} \geq 8 \sqrt{n+2}$. Now using that $2 \chi(r) \leq n \widetilde{V}(r)$, we have that the right hand side above tends to zero as $r \rightarrow \infty$. This induces that $\lim _{\sup _{r \rightarrow \infty}} r^{-n} \widetilde{V}(r)=0$, which is a contradiction.

We should remark that there exists a proof to Perelman's result by Hamilton via his singularity analysis of ancient solutions. The interested reader can find the details of Hamilton's argument in [CLN]. It is interesting to find out if Proposition 2.1 can be shown for any ancient solutions with nonnegative Ricci curvature.

\section{Optimal Transport and LSIs}

In this section, we will work with Riemannian manifolds $(M, g)$ endowed with a reference probability measure $e^{-V} d \Gamma$ where the potential $V \in C^{2}(M)$ verifies a curvature-dimension bound of the type $C(K, \infty)$ with $K \in \mathbb{R}$, i.e.,

$$
R_{i j}+V_{i j} \geq K g_{i j} .
$$

Here $d \Gamma$ is the volume measure associated to $(M, g)$. This section is devoted to collect several results present in the literature [Vi2]. A Riemannian manifold in this section refers to a smooth, complete connected finite-dimensional Riemannian manifold distinct from a 
point, equipped with a smooth metric tensor. Let us assume that the reference measure is normalized by

$$
\int_{M} e^{-V} d \Gamma=1
$$

Consider the positive solution $\rho$ to the Fokker-Planck equation

$$
\frac{\partial \rho}{\partial t}-\operatorname{div}(\rho \nabla(\log \rho+V))=0
$$

Let $\xi=\log \rho+V$. It is easy to see that

$$
\left(\frac{\partial}{\partial t}-\Delta\right) \xi=\langle\nabla \xi, \nabla \log \rho\rangle .
$$

Let us define the Boltzmann relative entropy functional, called also Nash entropy, as

$$
H_{V}(\rho) \doteqdot \int_{M} \rho \xi d \Gamma
$$

We have immediately the following dissipation of the Boltzmann relative entropy functional,

$$
\frac{d}{d t} H_{V}(\rho(t))=-\int_{M}|\nabla \xi|^{2} \rho d \Gamma \doteqdot-I_{V}(\rho(t)),
$$

where computations are made for smooth, fast-decaying at infinity for non-compact manifolds, solutions on $M$. This computation show us that these two quantities, the relative Boltzmann entropy $H_{V}(\rho)$ and the relative Fisher information $I_{V}(\rho)$ are intimately related at least for solutions of (3.1). However, as it was discovered in the case of $\mathbb{R}^{n}$, and in the case of a manifold in [BE, AMTU] for linear diffusions or in [CT, O, DoPi] for nonlinear diffusions, this relation is really through functional inequalities, see also [OV].

Related to these functionals, there is another quantity that is involved in these inequalities: the Euclidean Wasserstein distance between any two probability measures $\nu_{0}, \nu_{1}$ on the manifold $M$, i.e.,

$$
W_{2}\left(\nu_{0}, \nu_{1}\right) \doteqdot \inf \left\{\int_{M \times M} r^{2}(x, y) d \theta(x, y) ; \theta \in \Theta\left(\nu_{0}, \nu_{1}\right)\right\}^{1 / 2} ;
$$

where $\Theta\left(\nu_{0}, \nu_{1}\right)$ is the set of probability measures on $M \times M$ having marginals $\nu_{0}$ and $\nu_{1}, r(x, y)$ is the Riemannian distance between $x$ and $y$. This distance is well defined for probability measures $\nu_{0}$ and $\nu_{1}$ with second moment bounded, $\mathcal{P}_{2}(M)$, and metrizes the weak convergence of measures in the sense of [Vi2, Definition 6.7, Theorem 6.8]. The expression "second moment bounded" refers to the fact that the squared distance function $r^{2}(x)$ is integrable against the measures $\nu_{0}$ and $\nu_{1}$. It worths to mention that the curvaturedimension bound $C(K, \infty)$ with $K>0$ implies that the second moment of the reference measure (actually, all moments) $e^{-V}$ is bounded, see [Vi2, Theorem 18.11].

Recently, several authors [S, LV] based on early works [Mc, CMS], see [Vi2, Chapter 17] for a whole account of the history, have characterized curvature-dimension bounds in terms of the displacement convexity of the Boltzmann relative entropy functional. The notion of displacement convexity refers to convexity along pathes of minimal transport distance $W_{2}$ in the set of probability measures $\mathcal{P}_{2}(M)$. An expression of the convexity of these functionals are the so called HWI inequalities, named in this way since they involved the three functionals $H_{V}(\rho), I_{V}(\rho)$ and $W_{2}$. In the following, we will work with measures absolutely continuous against volume measure and we identify the measures with their densities for notational convenience. The main results we need are the following: 
Theorem 3.1. ([Vi2, Corollary 20.13] and [BE]) Let $M$ be a Riemannian manifold equipped with a reference measure $e^{-V} d \Gamma$ where the potential $V \in C^{2}(M)$ verifies a curvaturedimension bound of the type $C(K, \infty)$ with $K \in \mathbb{R}$. Then, for any given $\nu \in \mathcal{P}_{2}(M)$ absolutely continuous with respect to volume measure $d \Gamma$ with density $\rho$, it holds the HWI inequality:

$$
H_{V}(\rho) \leq W_{2}\left(\rho, e^{-V}\right) \sqrt{I_{V}(\rho)}-\frac{K}{2} W_{2}\left(\rho, e^{-V}\right)^{2} .
$$

As a consequence, we have that whenever $K>0$, the following LSI follows

$$
H_{V}(\rho) \leq \frac{1}{2 K} I_{V}(\rho) .
$$

The HWI inequalities were originally introduced in $[\mathrm{OV}]$ and used in other models in nonlinear PDEs in [CMV]. Later, they were generalized to compact manifolds in [LV] and in this generality in [Vi2]. To see that the LSI inequality follows from the HWI inequality it suffices to consider the right-hand side of the HWI inequality as a function of $W_{2}$ and maximize that function.

Let us remark that some proofs of the LSI inequality use the Fokker-Planck dynamics (3.1), called the Bakry-Emery stragegy, but the referred functional proof through the HWI inequalities allows to overcome discussions on integrability issues and the decay at infinity for non-compact manifolds of solutions to (3.1). In fact, a direct application of the LSI on (3.2) gives the exponential decay of the Boltzmann relative entropy functional for solutions of (3.1) with initial density in $\mathcal{P}_{2}(M)$ in case $C(K, \infty)$ with $K>0$ holds, i.e., given a solution $\rho(t)$ of $(3.1)$ then

$$
H_{V}(\rho(t)) \leq H_{V}(\rho(0)) e^{-2 K t} \quad \text { for all } t \geq 0 .
$$

Nevertheless, let us remind the reader that assuming all integrability and behavior at the infinity are met for all integration by parts below, we can obtain the evolution of the relative Fisher information (see also [BE, AMTU, Vi1, Vi2] for these computations). To take the time derivative of $I_{V}(\rho(t))$ note the Bochner type formula

$$
\left(\frac{\partial}{\partial t}-\Delta\right)|\nabla \xi|^{2}=-2 \xi_{i j}^{2}+2\langle\nabla(\langle\nabla \xi, \nabla \log \rho\rangle), \nabla \xi\rangle-2 R_{i j} \xi_{i} \xi_{j} .
$$

Using the above formula we have that

$$
\begin{aligned}
\frac{d}{d t} I_{V}(\rho(t))= & \int_{M}\left(\Delta|\nabla \xi|^{2}\right) \rho+|\nabla \xi|^{2} \operatorname{div}(\nabla \rho+\rho \nabla V) d \Gamma \\
& +\int_{M}\left(-2 \xi_{i j}^{2}+2\langle\nabla(\langle\nabla \xi, \nabla \log \rho\rangle), \nabla \xi\rangle-2 R_{i j} \xi_{i} \xi_{j}\right) \rho d \Gamma .
\end{aligned}
$$

Since

$$
\begin{aligned}
\int_{M}\langle\nabla(\langle\nabla \xi, \nabla \log \rho\rangle), \nabla \xi\rangle \rho d \Gamma= & \int_{M}\left\langle\nabla\left(|\nabla \xi|^{2}-\langle\nabla V, \nabla \xi\rangle\right), \nabla \xi\right\rangle \rho d \Gamma \\
= & \int_{M}\left\langle\left\langle\nabla|\nabla \xi|^{2}, \nabla \rho\right\rangle+\left\langle\nabla|\nabla \xi|^{2}, \nabla V\right\rangle \rho d \Gamma\right. \\
& -\int_{M}\langle\nabla\langle\nabla V, \nabla \xi\rangle, \nabla \xi\rangle \rho \Gamma
\end{aligned}
$$


we arrive at

$$
\begin{aligned}
\frac{d}{d t} I_{V}(\rho(t))= & \int_{M}\left(-2 \xi_{i j}^{2}-2 R_{i j} \xi_{i} \xi_{j}\right) \rho d \Gamma \\
& +\int_{M}\left\langle\nabla|\nabla \xi|^{2}, \nabla V\right\rangle \rho-2\langle\nabla\langle\nabla V, \nabla \xi\rangle, \nabla \xi\rangle \rho d \Gamma \\
= & \int_{M}\left(-2 \xi_{i j}^{2}-2\left(R_{i j}+V_{i j}\right) \xi_{i} \xi_{j}\right) \rho d \Gamma .
\end{aligned}
$$

As a consequence, due to the curvature dimension bound $C(K, \infty)$, we have

$$
\frac{d}{d t} I_{V}(\rho(t)) \leq-2 K \int_{M}|\nabla \xi|^{2} \rho d \Gamma
$$

and thus,

$$
I_{V}(\rho(t)) \leq I_{V}(\rho(0)) e^{-2 K t} \quad \text { for all } t \geq 0 .
$$

\section{Main Result and Applications}

Now, let us come back to the precise situation we have, the case of a shrinking soliton, and prove the main Theorem 1.1. Let us define the potential $V=f+\frac{n}{2} \log (4 \pi \tau)$ for the fixed time slice of the shrinking Riemannian manifold soliton $(M, g)$ at time $\tau$. Lemma 2.1 and Corollary 2.1 implies that $e^{-V}$ is a well defined probability measure. Moreover, we deduce from the soliton definition (1.2) that this reference measure verifies the $C\left(\frac{1}{2 \tau}, \infty\right)$ condition. Therefore, Theorem 3.1 implies that for any probability density of the form

$$
\rho(x)=\frac{e^{-\psi(x)}}{(4 \pi \tau)^{\frac{n}{2}}}
$$

with second moment bounded, we get the LSI

$$
H_{V}(\rho) \leq \tau I_{V}(\rho) .
$$

Using now the soliton equation (2.3), we deduce:

$$
\begin{aligned}
I_{V}(\rho) & =\int_{M}\left(|\nabla \psi|^{2} \rho+2\langle\nabla f, \nabla \rho\rangle+|\nabla f|^{2} \rho\right) d \Gamma \\
& =\int_{M}\left[|\nabla \psi|^{2} \rho+\left(-2 \Delta f+|\nabla f|^{2}\right) \rho\right] d \Gamma \\
& =\int_{M}\left[|\nabla \psi|^{2}+S+\frac{f+\mu_{s}-n}{\tau}\right] \rho d \Gamma .
\end{aligned}
$$

Thus, the LSI inequality is equivalent to

$$
\int_{M}\left[\tau\left(|\nabla \psi|^{2}+S\right)+\psi-n\right] \rho d \Gamma \geq-\mu_{s},
$$

for all densities $\rho$ with bounded second moment for the shrinking soliton, with $\mu_{s}$ characterized by Corollary 2.1.

Now recall Perelman's entropy functional

$$
\mathcal{W}\left(g^{\tau}, u, \tau\right) \doteqdot \int_{M}\left[\tau\left(|\nabla \psi|^{2}+S\right)+\psi-n\right] u d \Gamma_{\tau}
$$


is defined for $u=\frac{e^{-\psi}}{(4 \pi \tau)^{n / 2}}$ with $\int_{M} u d \Gamma_{\tau}=1$. Theorem 1.1 implies that for $\left(M, g^{\tau}\right)$, $W\left(g^{\tau}, u, \tau\right) \geq-\mu_{s}$. Namely Perelman's $\mu$-invariant

$$
\mu\left(g^{\tau}, \tau\right) \doteqdot \inf _{\int_{M} u=1} \mathcal{W}\left(g^{\tau}, u, \tau\right)
$$

is bounded from below by $-\mu_{s}$. From (2.3) it is easy to see that

$$
\tau\left(2 \Delta f-|\nabla f|^{2}+S\right)+f-n=-\mu_{s} .
$$

Hence $u=\frac{e^{-f}}{(4 \pi \tau)^{n / 2}}$ is the minimizer for Perelman's $\mu(g, \tau)$, cf. [P, Remark 3.2]. This shows that the inequality of Theorem 1.1 is sharp. Summarizing, we have that

Corollary 4.1. Let $(M, g, f)$ be a gradient shrinking soliton satisfying (1.1). Then

$$
\mu(g, 1)=-\mu_{s} .
$$

Remark 4.2. When $f=$ constant, $(M, g)$ is a Einstein manifold with $\operatorname{Ric}_{M}=\frac{1}{2} g_{M}$. In this case we obtain a log-Sobolev inequality for $S=\frac{n}{2}$ and

$$
\mu_{s}=\frac{n}{2}-\log (V(M))+\frac{n}{2} \log (4 \pi)
$$

where $V(M)$ is the volume of $\left(M, g_{M}\right)$. The $\mu$-invariant was computed in $[\mathrm{CHI}]$ for many examples of four manifolds.

When $M=\mathbb{R}^{n}$ with $f=\frac{1}{4}|x|^{2}$, direct calculation shows that $\mu_{s}=0$. Hence the classical logarithmic Sobolev inequality of Stam-Gross is a special case.

Recall here that a solution of Ricci flow is called $\kappa$ non-collapsed, if for any $\left(x_{0}, t_{0}\right)$ and $r \geq 0$, such that on $P\left(x_{0}, t_{0}, r\right)=B_{g\left(t_{0}\right)}\left(x_{0}, r\right) \times\left[t_{0}-r^{2}, t_{0}\right],|R m|(x, t) \leq r^{-2}$, then $V_{g\left(t_{0}\right)}\left(x_{0}, r\right) \geq \kappa r^{n}$. Here $V_{g\left(t_{0}\right)}\left(x_{0}, r\right)$ is the volume of $B_{g\left(t_{0}\right)}\left(x_{0}, r\right)$ with respect to $g\left(t_{0}\right)$. Perelman [P, Theorem 1.1] implies the following volume non-collapsing result for gradient shrinking solitons.

Corollary 4.3. Let $(M, g, f)$ be a gradient shrinking soliton satisfying (1.1). Then there exists a $\kappa=\kappa\left(\mu_{s}\right)>0$ such that if in a ball $B\left(x_{0}, 1\right), \mid$ Ric $\mid \leq 1$, then $V\left(x_{0}, 1\right) \geq \kappa$. In particular, if the Ricci curvature is bounded on $M$ which is noncompact, then $M$ has at least linear volume growth.

Proof. Follows from Theorem 1.1 and Section 4 of [P]. See also [Chow, et al1, To].

In $[\mathrm{Na}]$ there is a related result asserting the $\kappa$-noncollapsing of gradient shrinking solitions with bounded curvature, in the sense defined right above the corollary. The conclusion in above corollary appears stronger since it only requires global lower bound on the scalar curvature and the local bound of the Ricci curvature over the ball, for a fixed time-slice only.

When $\operatorname{Ric}\left(M, g_{\tau=1}\right) \geq 0$ and is bounded, one can derive the logarithmic Sobolev inequality for all scales. This is done in the following two propositions.

Proposition 4.1 (Scale $>1)$. Let $(M, g)$ be a gradient shrinking soliton satisfying (1.1). Assume that Ric $\geq 0$. Then, there exists positive $\delta=\delta(M)<1$ such that for any $\sigma>1$,

$$
\int_{M}\left[\sigma\left(|\nabla \tilde{\psi}|^{2}+S\right)+\tilde{\psi}-n\right] \frac{e^{-\tilde{\psi}}}{(4 \pi \sigma)^{\frac{n}{2}}} d \Gamma \geq-\mu_{s}+\frac{n}{2}-\delta-\frac{n}{2} \log \left(\frac{n}{2 \delta}\right)
$$

for any $\tilde{\psi}$ satisfying that $\int_{M} e^{-\tilde{\psi}} /(4 \pi \sigma)^{\frac{n}{2}} d \Gamma=1$. 
Proof. Clearly only the nonflat case worths the proof (since the flat one is isometric to $\mathbb{R}^{n}$ ). By [N3, Proposition 1.1], for a nonflat gradient shrinking soliton, there exists $\delta=\delta(M, f)>$ 0 such that $S(x) \geq \delta$ for any $x \in M$. Let $\psi=\tilde{\psi}+\frac{n}{2} \log \sigma$. Then it is easy to see that

$$
\begin{aligned}
\int_{M}\left[\sigma\left(|\nabla \tilde{\psi}|^{2}+S\right)+\tilde{\psi}-n\right] \frac{e^{-\tilde{\psi}}}{(4 \pi \sigma)^{\frac{n}{2}}} d \Gamma= & \int_{M}\left(|\nabla \psi|^{2}+S+\psi-n\right) \frac{e^{-\psi}}{(4 \pi)^{\frac{n}{2}}} d \Gamma \\
& +(\sigma-1) \int_{M}\left(|\nabla \psi|^{2}+S\right) \frac{e^{-\psi}}{(4 \pi)^{\frac{n}{2}}} d \Gamma-\frac{n}{2} \log \sigma \\
\geq & -\mu_{s}+\delta(\sigma-1)-\frac{n}{2} \log \sigma,
\end{aligned}
$$

where we have used Theorem 1.1 in the last estimate. Since $\delta(\sigma-1)-\frac{n}{2} \log \sigma \geq \frac{n}{2}-\delta-$ $\frac{n}{2} \log \left(\frac{n}{2 \delta}\right)$, the claimed result follows.

From the proof, the following corollary is evident, observing that $S \geq 0$ for shrinking solitons, which is clear from [Ch], see also the appendix of [Yo].

Corollary 4.4. Let $(M, g)$ be a gradient shrinking soliton satisfying (1.1). Then for any $\sigma>1$,

$$
\int_{M}\left[\sigma\left(|\nabla \tilde{\psi}|^{2}+S\right)+\tilde{\psi}-n\right] \frac{e^{-\tilde{\psi}}}{(4 \pi \sigma)^{\frac{n}{2}}} d \Gamma \geq-\mu_{s}-\frac{n}{2} \log \sigma .
$$

Proposition 4.2 (Scale $<1)$. Assume that $0 \leq$ Ric $\leq A$. Then for any $0 \leq \sigma \leq 1$,

$$
\int_{M}\left[\sigma\left(|\nabla \tilde{\psi}|^{2}+S\right)+\tilde{\psi}-n\right] \frac{e^{-\tilde{\psi}}}{(4 \pi \sigma)^{\frac{n}{2}}} d \Gamma \geq-\mu_{s}-n A
$$

for any $\tilde{\psi}$ satisfying that $\int_{M} e^{-\tilde{\psi}} /(4 \pi \sigma)^{\frac{n}{2}} d \Gamma=1$.

Proof. Define

$$
\mu_{0}(g, \sigma) \doteqdot \inf _{\int_{M} u_{0}=1} \int_{M}\left(\sigma|\nabla \tilde{\psi}|^{2}+\tilde{\psi}-n\right) u_{0} d \Gamma
$$

with $u_{0}=e^{-\tilde{\psi}} /(4 \pi \sigma)^{\frac{n}{2}}$. Theorem 1.1 implies that $\mu_{0}(g, 1) \geq-\mu_{s}-n A$. Now for any $u_{0}$ which is compactly supported, let $u(x, t)$ be the heat equation solution with $u(x, 0)=u_{0}$. Then by the entropy monotonicity result in [N2], for $\sigma \leq 1$,

$$
\begin{aligned}
\int_{M}\left(\sigma|\nabla \tilde{\psi}|^{2}+\tilde{\psi}-n\right) \frac{e^{-\tilde{\psi}}}{(4 \pi \sigma)^{\frac{n}{2}}} & \geq \int_{M}\left(|\nabla \varphi|^{2}+\varphi-n\right) u(y, 1-\sigma) d \Gamma(y) \\
& \geq \mu_{0}(g, 1)
\end{aligned}
$$

where $u(y, 1-\sigma)=e^{-\varphi(y)} /(4 \pi(1-\sigma))^{\frac{n}{2}}$. This implies the claimed result.

The above two propositions imply that $\nu(g)>-\infty$, see section 7 for a definition, hence the strong $\kappa$-non-collapsing result for gradient shrinking solitons with bounded and nonnegative Ricci curvature as in $[\mathrm{P}]$ (see also [To] and [Chow, et al1]). For the general case without assuming Ric $\geq 0$, one can still obtain a logarithmic Sobolev for scales less than one, see Section 7 .

\section{EXPANDING SOLITONS}

Recall that $(M, g)$ is called a gradient expanding soliton if there exists $f$ such that

$$
R_{i j}+\frac{1}{2} g_{i j}=f_{i j}
$$


It is easy to show that

$$
\begin{aligned}
\Delta f & =S+\frac{n}{2} \\
S+|\nabla f|^{2}-f & =\mu_{e}
\end{aligned}
$$

for some constant $\mu_{e}$. As before we will eventually choose $\mu_{e}$ by the normalizing condition $\int_{M} e^{-f} /(4 \pi)^{n / 2} d \Gamma=1$. This will make $\mu_{e}$ a geometric invariant of $(M, g)$. T

Our first concern is about the behavior of the volume of balls $B(o, r)$ in $M$ for any given $o \in M$. Along this direction, Hamilton [H2] proved the following result:

Theorem 5.1. Let $(M, g)$ be a gradient expanding soliton has bounded nonnegative Ricci curvature. Then $(M, g)$ has maximum volume growth. Namely

$$
\liminf _{r \rightarrow \infty} \frac{V(o, r)}{r^{n}}>0
$$

For the exposition of this result please see [CLN, Proposition 9.46]. Let us remark that the assumption of uniform boundedness of the Ricci curvature is used in the proof to bound $\int_{\gamma} \operatorname{Ric}\left(\gamma^{\prime} \gamma^{\prime}\right)$ as in Section 2. Here, the limit always exists due to the Bishop-Gromov volume comparison. The limit of the quotient is called the asymptotic volume ratio. This compares sharply with the gradient shrinking solitons (cf. Proposition 2.1) and a result of Perelman $[\mathrm{P}]$ asserting that any non-flat ancient solution with bounded nonnegative curvature operator has zero asymptotic volume ratio. The result below is a generalization of the above result of Hamilton.

Proposition 5.1. Let $(M, g, f)$ be an gradient expanding soliton.

(1) If $S(x) \geq 0$ for any $x \in M$, without assuming any curvature bound, then for any $o \in M$, $r \geq r_{0}$.

$$
V(o, r) \geq V\left(o, r_{0}\right)\left(\frac{r+a}{r_{0}+a}\right)^{n}
$$

with $a=2 \sqrt{f(o)+\mu_{e}}$.

(2) Assume that $S(x) \geq-\beta$ for some constant $\beta>0$. Then for any $o \in M$ and $r \geq r_{0}$

$$
V(o, r) \geq V\left(o, r_{0}\right)\left(\frac{r+a}{r_{0}+a}\right)^{n-2 \beta}
$$

with $a=2 \sqrt{f(o)+\mu_{e}+\beta}$.

Proof. In the case (1), from the assumption and (5.3) we have that $f+\mu_{e} \geq 0$. Consider any minimizing geodesic $\gamma(s)$ from $o \in M$ a fixed point of $M$. Then (5.3) implies that for any $s$

$$
\left|\frac{d}{d s} f(\gamma(s))\right|^{2} \leq f+\mu_{e} .
$$

This implies, by the ODE comparison, that

$$
\left(2 \sqrt{f+\mu_{e}}\right)(\gamma(s)) \leq s+a
$$

where $a=2 \sqrt{f(o)+\mu_{e}}$, which then implies that

$$
\left|\frac{\partial f}{\partial r}\right|(\gamma(s)) \leq \frac{s}{2}+\frac{a}{2} .
$$


Now we integrate $(5.2)$ on $B(o, r)$ and have that

$$
\begin{aligned}
\frac{n}{2} V(o, r) & \leq \frac{n}{2} V(o, r)+\int_{B(o, r)} S d \Gamma \\
& =\int_{B(o, r)} \Delta f d \Gamma \\
& \leq \int_{\partial B(o, r)}\left|\frac{\partial f}{\partial r}\right|(y) d A(y)
\end{aligned}
$$

Using (5.4) we have that

$$
\frac{n}{2} V(o, r) \leq A(o, r)\left(\frac{r}{2}+\frac{a}{2}\right)
$$

The result follows by dividing the both side of the above by $V(o, r)$ and then integrating the resulting estimate on the interval $\left[r_{0}, r\right]$. The proof for the case $(2)$ is similar.

Remark 5.2. The estimates in both cases have the sharp power. To see this consider $M=N^{k} \times \mathbb{R}^{n-k}$ where $N$ is a compact Einstein manifold with $\operatorname{Ric}_{N}=-\frac{1}{2} g_{N}, \mathbb{R}^{n-k}$ is the Gaussian expanding soliton.

Now we derive the LSI for the expanders. To make sure that the integral $\int_{M} e^{-f} d \Gamma$ is finite we have to make an assumption that there exists some $\epsilon>0$,

$$
f_{i j}=\frac{1}{2} g_{i j}+R_{i j} \geq \epsilon g_{i j} .
$$

Under this assumption, it is easy to see that

$$
f(x) \geq \frac{\epsilon}{4} r^{2}(x)-C
$$

for some $C=C(M, f)$. Since $R_{i j} \geq-\frac{1}{2} g_{i j}$, the volume $V(o, r) \leq \exp (A(r+1))$ for some $A=A(n)$. This together with the lower estimate above ensures that the integral $\int_{M} e^{-f} d \Gamma$ is finite, see also [WW]. Notice that under our assumption (5.5), as in the proof of Proposition 5.1 we have that

$$
f(x) \leq\left(\frac{r(x)}{2}+b\right)^{2}
$$

for some $b=b(M, f)$. This ensures the finiteness of the integral

$$
\int_{M}\left(|\nabla f|^{2}+|\Delta f|+|S|\right) \frac{e^{-f}}{(4 \pi)^{n / 2}} d \Gamma .
$$

Note that (5.2) and (5.3) implies that

$$
2 \Delta f-|\nabla f|^{2}-3 S+f-n=-\mu_{e} .
$$

Integrating (5.6), we have that

$$
\int_{M}\left(|\nabla f|^{2}-3 S+f-n\right) \frac{e^{-f}}{(4 \pi)^{n / 2}} d \Gamma=-\mu_{e}
$$

It is clear that assumption (5.5) is trivially satisfied for the case that $M$ has non-negative Ricci curvature.

Assume in the rest of this section that Ric $\geq 0$, let us define the potential $V=f-\frac{n}{2} \log (4 \pi)$. Previous arguments imply that the reference measure $e^{-V}$ is a well defined probability measure. Moreover, we deduce from the soliton definition (5.1) and being Ric $\geq 0$ that this 
reference measure verifies the $C\left(\frac{1}{2}, \infty\right)$ condition. Therefore, Theorem 3.1, together with a similar calculation as before, implies the following LSI inequality.

Theorem 5.3. Assume that $(M, g, f)$ is gradient expanding soliton with $\mathrm{Ric} \geq 0$. Then for any $\rho(x)=e^{-\psi(x)} /(4 \pi)^{n / 2}$ with $\int_{M} \rho(x) d \Gamma(x)=1$, we have that

$$
\int_{M}\left(|\nabla \psi|^{2}-3 S+\psi-n\right) \rho d \Gamma \geq-\mu_{e} .
$$

Here $\mu_{e}$, as before, is a geometric invariant (in the sense of Section 2), which is the same for two isometric metrics. One can write in the dynamic form by considering the family of metrics $g(\tau)$ (in this case with $g(1)$ being the original metric, and $0<\tau<\infty$ ) generated by the diffeomorphisms, as for the shrinking solitons case described in the introduction. Since it is the same inequality by re-scaling we omit its full statement. Note that in the left hand side of (5.7) an equivalent integrand is

$$
\tau\left(2 \Delta \psi-|\nabla \psi|^{2}-3 S\right)+\psi-n .
$$

This expression also showed itself up in a differential Harnack or Li-Yau-Hamilton type calculation, in a recent preprint of Cao and Hamilton $[\mathrm{CH}]$, where however the nonnegativity of the curvature operator is required. It is certainly interesting to explore the connections between the LSI here and the Li-Yau-Hamilton type estimate for Ricci flow solution.

Corollary 5.4. Let $(M, g)$ be an expanding soliton as in Theorem 5.3. Then $M$ is diffeomorphic to $\mathbb{R}^{n}$.

Proof. First it is easy to see that $M$ is of finite topological type. This follows from the observation that $f$ is a proper function and has no critical point outside a compact subset [FMZ], since for any $x \in M$ and $\gamma(s)$ a minimizing geodesic jointing $o \in M$, a fixed point, to $x$, with $f(\gamma(0))=o$ and $f\left(\gamma\left(s_{0}\right)\right)=x$

$$
\begin{aligned}
f^{\prime}\left(\gamma\left(s_{0}\right)\right) & =f^{\prime}(\gamma(0))+\int_{0}^{s_{0}} f^{\prime \prime}(\gamma(s)) d s \\
& \geq \frac{s_{0}}{2}+f^{\prime}(\gamma(0)) .
\end{aligned}
$$

The conclusion follows from the uniqueness of the critical point along with the strict convexity of $f$.

\section{Gradient steady solitons}

Now we consider the gradient steady solitons. Recall that a gradient steady soliton $(M, g)$ has a potential function $f$ satisfying that

$$
R_{i j}=f_{i j}
$$

It was shown in [H1] that

$$
|\nabla f|^{2}+S=\lambda
$$

for some $\lambda$. Similar as before there is a solution to Ricci flow $g(\tau)$ associated with the gradient steady soliton $(M, g, f)[\mathrm{CLN}]$. We first need the following lemma to ensure the finiteness of $\int_{M} e^{-f} d \Gamma$ and other integrals later involved, under some geometric assumptions. 
Lemma 6.1. Let $(M, g, f)$ be a gradient steady soliton. Assume that there exists a point $o \in M$ such that $S(o)=\max _{M} S$ and either $\operatorname{Ric}(x)>0$ for all $x \in M$, or Ric $\geq 0$ and

$$
\limsup _{x \rightarrow \infty} S(x)<\max _{M} S
$$

Then $o$ is a minimum of $f$ and there exists $\delta>0$ and $C=C(M, f)$ so that

$$
f(x) \geq \delta r(x)-C \text {. }
$$

Here $r(x)$ is the distance function to o. In particular, $M$ is diffeomorphic to $\mathbb{R}^{n}$ in the case Ric $>0$ and of finite topological type in the case Ric $\geq 0$.

Proof. For the first case, it was shown in [H1, Theorem 20.1] that $o$ is the unique minimum of $f$. Note that the argument there actually requires $\mathrm{Ric}>0$ even though it was not stated; it is also necessary, as shown by easy examples. Note that for any geodesic $\gamma(s)$ from $o$, we have that

$$
\frac{d^{2}}{d s^{2}}\left(f(\gamma(s))=\operatorname{Ric}\left(\gamma^{\prime}, \gamma^{\prime}\right)>0\right.
$$

Hence we have for any $s_{0}>0, \frac{d}{d s}\left(f\left(\gamma\left(s_{0}\right)\right)\right)>0$. Then $f(\gamma(s)) \geq \frac{d}{d s}\left(f\left(\gamma\left(s_{0}\right)\right)\right)\left(s-s_{0}\right)+$ $f\left(\gamma\left(s_{0}\right)\right)$, which implies the desired lower estimate.

For the second case, the assumption already excludes the Ricci flat situation, on which clearly (6.3) fails for $f$ being a constant. We first claim that under the assumption on the behavior of $S$ at the infinity, $S(o)=\lambda$. Suppose it is not true, then $\max _{M} S<\lambda$ and $|\nabla f|^{2} \geq \lambda-\max _{M} S$. Let $\sigma(u)$ be an integral curve of $\nabla f$ passing $o$ with $\sigma(0)=o$. Direct calculation shows that $\frac{d}{d u}\left(|\nabla f|^{2}(\sigma(u))\right)=2 \operatorname{Ric}(\nabla f, \nabla f)(\sigma(u)) \geq 0$. This shows that $|\nabla f|^{2}(\sigma(u))=|\nabla f|^{2}(\sigma(0))$ for $u \leq 0$ since $|\nabla f|^{2}$ has its minimum at $o$. Hence we have that $S(\sigma(u))=\max _{M} S$ for all $u \leq 0$. However since $-f(\sigma(u))=-f(\sigma(0))+\int_{u}^{0}|\nabla f|^{2} d u=$ $-f(\sigma(0))-u|\nabla f|^{2}(\sigma(0)) \rightarrow+\infty$ as $u \rightarrow-\infty$ we can conclude that $\sigma(u) \rightarrow \infty$. This is a contradiction with the assumption that $\lim _{\sup _{x \rightarrow \infty}} S(x)<\max _{M} S$. Hence we have that $\lambda=\max _{M} S$ which implies $\nabla f=0$ at $o$ and

$$
\liminf _{x \rightarrow \infty}|\nabla f|^{2} \geq 2 \eta^{2} \doteqdot \lambda-\limsup _{x \rightarrow \infty} S(x)>0 .
$$

By considering any minimizing geodesic $\gamma(s)$ emitting from $o$ and the fact $\frac{d}{d s}(f(\gamma(0)))=0$ and $\frac{d^{2}}{d s^{2}}(f(\gamma(s))) \geq 0$, it is clear that $o$ is the minimal point of $f$ and $\langle\nabla f, \nabla r\rangle(x) \geq 0$ for any $x \in M \backslash\{o\}$. Let $R_{0}$ be such that $|\nabla f|^{2}(x) \geq \eta^{2}$ for all $x \in M \backslash B\left(o, R_{0}\right)$. Consider again an integral curve $\sigma(u)$ passing $x$. Since $|\nabla f|$ is bounded and $M$ is complete, the curve is defined for all $-\infty<u<+\infty$. Notice that $\sigma(u) \in B(o, r(x))$ for all $u \leq 0$ and

$$
f(\sigma(0))-f(\sigma(u))=\int_{u}^{0}|\nabla f|^{2} d u \geq(-u) \eta^{2}
$$

as along as $\sigma(u) \in M \backslash B\left(o, R_{0}\right)$. From this we infer that there exist some $u_{0}$ such that $\sigma\left(u_{0}\right) \in B\left(o, R_{0}\right)$. On the other hand

$$
f(x)=f\left(\sigma\left(u_{0}\right)\right)+\int_{u_{0}}^{0}|\nabla f|^{2} d u \geq f\left(\sigma\left(u_{0}\right)\right)+\eta \int_{u_{0}}^{0}\left|\sigma^{\prime}(u)\right| d u \geq f\left(\sigma\left(u_{0}\right)\right)+\eta d\left(x, \sigma\left(u_{0}\right)\right) .
$$

This implies the desired lower estimate. The final conclusion follows easily from the above estimate on $|\nabla f|$ and the convexity of $f$. 
Remark 6.1. If the sectional curvature of $(M, g)$ is nonnegative, one can show that the claim of the lemma holds under the assumption that $S(o)=\max _{M} S$, as far as $M$ does not admit any flat factor $\mathbb{R}^{k}$. The reason is the following. First if the claimed result fails, one can conclude that $f_{i j}$ has an eigenvector corresponding to the zero eigenvalue somewhere. Note that for the associated Ricci flow, the function $f(x, \tau)$, defined as the pull back via the diffeomorphism generated by $\nabla f$, satisfies the heat equation (cf. [CLN] for details). Then the result follows from the strong tensor maximum principle and a splitting theorem of noncompact manifolds proved in [N1].

In the both cases $o$ is a minimum point of $f$ and $\lambda$ is a geometric invariant, namely $\max _{x \in M} S(x)$. Also we have seen that both $|\nabla f|$ and $|\Delta f|$ are bounded. We normalize $f$ so that $\int_{M} e^{-f} d \Gamma=1$. Integration by parts gives the following weighted Poincaré inequality.

Proposition 6.1. Let $(M, g, f)$ be a gradient steady soliton. Then for any compact supported smooth function $u=e^{-\psi}$ with $\int_{M} u d \Gamma=1$, we have that

$$
\int_{M}\left(|\nabla \psi|^{2}-3 S\right) u d \Gamma \geq-\lambda .
$$

Proof. The proof follows from the following simple calculation:

$$
\begin{aligned}
\int_{M}\left(|\nabla \psi|^{2}-3 S\right) u & =\int_{M}\left(|\nabla \psi|^{2}-2\langle\nabla \psi, \nabla f\rangle+|\nabla f|^{2}+2 \Delta f-|\nabla f|^{2}-3 S\right) u \\
& \geq \int_{M}\left(2 \Delta f-|\nabla f|^{2}-3 S\right) u=-\lambda
\end{aligned}
$$

for all normalized $u$.

This is a sharp inequality, at least it is so under the assumption of Lemma 6.1, since for this case the equality holds when $u=e^{-f}$. An equivalent form is that

$$
\int_{M}\left(4|\nabla \varphi|^{2}-3 S \varphi^{2}\right) d \Gamma \geq-\lambda \int_{M} \varphi^{2} d \Gamma
$$

for any $\varphi \in L^{2}(M)$. The weighted Poincaré inequality and its geometric meanings have recently been studied in $[\mathrm{LW}]$.

\section{An analogue of THE C-THEOREM-NONNEGATIVITY OF $\mu_{s}$ AND $\mu_{e}$}

For the re-normalization group flow, there exists the so-called central charge $c(t)$ invariant $[\mathrm{Z}]$ for the flow such that $c(t)$ is monotone non-increasing in $t$. Moreover $c(t)$ is always nonnegative. For Ricci flow, there are Perelman's monotonic quantities such as the $\mathcal{W}(g, \sigma, f)$-entropy, defined as

$$
\mathcal{W}(g, \sigma, \varphi) \doteqdot \int_{M}\left(\sigma\left(|\nabla \varphi|^{2}+S\right)+\varphi-n\right) u d \Gamma
$$

for any $u=e^{-\varphi} /(4 \pi \sigma)^{\frac{n}{2}}$ with $\int_{M} u=1$, and associated $\mu(g, \sigma) \doteqdot \inf _{\int_{M} u=1} \mathcal{W}(g, \sigma, \varphi)$, $\nu(g) \doteqdot \inf _{\sigma>0} \mu(g, \sigma)$ invariants, as well as the so-called reduced volume. The quantity $\mathcal{W}(g, \sigma, \varphi), \mu(g, \sigma)$ and $\nu(g)$ may not be finite when $M$ is not compact. Proposition 4.1 and Proposition 4.2 ensures that is the case for the shrinkers with bounded nonnegative Ricci curvature. The reduced volume $[\mathrm{P}]$ is always nonnegative by the definition. But it is monotone non-decreasing instead of non-increasing in $t$ (along the flow). Utilizing the sharp LSI's proved for the shrinkers and expanders we shall show in this section that 
the logarithmic Sobolev constants $\mu_{s}$ and $\mu_{e}$ are nonnegative, at least for the gradient shrinking/expanding solitons (with some mild assumptions on the Ricci curvature). In view of the monotonicity of the entropy, and the fact that the gradient shrinking solitons often arises at the singularity, one can view the monotonicity of the entropy together with the result proved here as an analogue of the c-theorem. Namely, for the solution to the Ricci flow, one can view $-\mu(g, \tau)$ as the analogue of the $c(t)$-invariant. Perelman's entropy formula concludes that it is monotone non-increasing. Our result concludes that $-\mu(g, 1)=\mu_{s}$ and it is nonnegative. One should note that if the $\nu(g(t))$ invariant of Perelman [P, Section 3] is well-defined/finite, unfortunately this is not always the case, then at least for the compact manifolds, the $-\nu(g(t))$ would be nonnegative and non-increasing along the Ricci flow.

We shall show two results on the sign of the invariants $\mu_{s}$ and $\mu_{e}$. The case of $\mu_{e}$ is an easy application of a rigidity result in [N2].

Proposition 7.1. Let $(M, g, f)$ be a gradient expanding soliton with $\mathrm{Ric} \geq 0$. Then $\mu_{e} \geq 0$. If $\mu_{e}=0$ then $(M, g)$ must be isometric to $\mathbb{R}^{n}$.

Proof. Assuming that $\mu_{e} \leq 0$, Theorem 5.3 then implies that

$$
\int_{M}\left(|\nabla \psi|^{2}+\psi-n\right) \rho d \Gamma \geq 0
$$

Then by [N2, Theorem 1.4], one can see a detailed account in [Chow, et al1, pages 314-333], we can conclude that $(M, g)$ is isometric to $\mathbb{R}^{n}$, on which $\mu_{e}=0$.

Similar result holds for gradient shrinking solitons. For that we have to assume that the curvature tensor of $(M, g)$ is bounded.

Theorem 7.1. Let $(M, g)$ be a gradient shrinking soliton with bounded curvature. Let $f$ be the normalized potential function as before, then $\mu_{s} \geq 0$.

Remark 7.2. After the appearance of our paper, Yokota [Yo] generalized the above result by assuming only the lower bound of Ricci curvature. The proof makes uses of Perelman's reduced volume.

Proof. We first prove the result under the extra assumption that Ric $\geq 0$. Recall from the introduction that there is an associated solution $g(t)$ (with $-\infty<t<0, t=\eta-1$ ) to Ricci flow generated by pulling back the metric via the diffeomorphisms generated by the vector field $\nabla f$. The original metric $g$ corresponds to the one $g(-1)$ (meaning $t=-1$ ). Proposition 4.1 and Proposition 4.2 imply that $\mu(g(-1), \sigma)$ and $\nu(g(-1))$ are finite. Since $g(t)$ is just the re-scale of $g(-1)$, we have that for any $-\infty<t<0, \mu(g(t), \sigma), \nu(g(t))$ are also finite. Now let $H\left(y, t ; x, t_{0}\right)$ (with $t<t_{0}<0$ ) be the (minimal) positive fundamental solution to the conjugate heat equation:

$$
\left(-\frac{\partial}{\partial t}-\Delta_{y}+S(y, t)\right) H\left(y, t ; x, t_{0}\right)=0
$$

being the $\delta_{x}(y)$ at $t=t_{0}$. By a result of Perelman [P, Corollary 9.3], see also [CTY, N4], we know that

$$
v_{H}(y, t) \doteqdot\left(t_{0}-t\right)\left(2 \Delta \varphi-|\nabla \varphi|^{2}+S\right)+\varphi-n \leq 0
$$

with $H\left(y, t ; x, t_{0}\right)=e^{-\varphi(y, t)} /\left(4 \pi\left(t_{0}-t\right)\right)^{\frac{n}{2}}$. This implies in particular

$$
\mu\left(g(-1), t_{0}+1\right) \leq \int_{M} v_{H}(y,-1) H(y,-1) d \Gamma_{g(-1)} \leq 0 .
$$


Here to ensure the inequality $v_{H} \leq 0$ the extra assumption that the curvature tensor of $M$ is uniformly bounded is needed [CTY].

On the other hand Theorem 1.1 asserts that $\mu(g(-1), 1) \geq-\mu_{s}$. The result would follow if we show that $\mu\left(g(-1), t_{0}+1\right) \rightarrow \mu(g(-1), 1)$ as $t_{0} \rightarrow 0$. For $t_{0, i} \rightarrow 0$, consider minimizers $\varphi_{i}$ of $\mathcal{W}\left(g(-1), 1+t_{0, i}, \varphi\right)$ (for simplicity we write $g(-1)$ back to $g$ from now on). Let $\sigma_{i}=1+t_{0, i} \rightarrow 1$. We assume that $\frac{1}{2} \leq \sigma_{i} \leq 1$. By Proposition 4.2 and the above we have that

$$
0 \geq \mu\left(g, \sigma_{i}\right) \geq-\mu_{s}-n A .
$$

Write $w_{i}=e^{-\varphi_{i} / 2}$. Then, essentially from definition, the $w_{i} \in W^{1,2}(M)$. The EulerLagrangian equation is

$$
-4 \sigma_{i} \Delta w_{i}+\sigma_{i} S w_{i}-n w_{i}-2 w_{i} \log w_{i}=\mu\left(g, \sigma_{i}\right) w_{i}
$$

for $\int_{M} w_{i}^{2}=\left(4 \pi \sigma_{i}\right)^{\frac{n}{2}} \leq(4 \pi)^{\frac{n}{2}}$. Integrating over $M$ we have that

$$
4 \sigma_{i} \int_{M}\left|\nabla w_{i}\right|^{2}=\mu\left(g, \sigma_{i}\right)\left(4 \pi \sigma_{i}\right)^{\frac{n}{2}}+\int_{M}\left(w_{i}^{2} \log w_{i}^{2}+n w_{i}^{2}-\sigma_{i} S w_{i}^{2}\right)
$$

which implies

$$
4 \sigma_{i} \int_{M}\left|\nabla w_{i}\right|^{2} \leq \int_{M} w_{i}^{2} \log w_{i}^{2}+n(4 \pi)^{\frac{n}{2}}
$$

On the other hand, writing $w_{i}^{2} /\left(4 \pi \sigma_{i}\right)^{\frac{n}{2}}=e^{-\tilde{\psi}} / \pi^{\frac{n}{2}}$ and using that $\mathcal{W}\left(g, \frac{1}{4}, \tilde{\psi}\right) \geq \mu\left(g, \frac{1}{4}\right)$,

$$
\int_{M}\left|\nabla w_{i}\right|^{2} \geq\left(4 \pi \sigma_{i}\right)^{\frac{n}{2}} \mu\left(g, \frac{1}{4}\right)-\frac{n A}{4}\left(4 \pi \sigma_{i}\right)^{\frac{n}{2}}+\int_{M} w_{i}^{2} \log w_{i}^{2}-\frac{n}{2} \log \left(4 \pi \sigma_{i}\right) .
$$

Combining with $(7.2)$, one can find $C=C(A, n)$ such that

$$
\int_{M}\left|\nabla w_{i}\right|^{2} \leq C(A, n)
$$

which implies that $\left\|w_{i}\right\|_{W^{1,2}(M)}$ is uniformly bounded. It then implies that $w_{i} \rightarrow w_{\infty}$ in the the dual norm of $W^{1,2}(M)$ and strongly in $L^{2}(M)$, for some $w_{\infty} \in W^{1,2}(M)$. Due to the bound $\mu\left(g, \sigma_{i}\right)$ we may also assume that $\mu\left(g, \sigma_{i}\right) \rightarrow \mu_{\infty}(g)$. Clearly $\mu_{\infty}(g) \leq 0$. It is evident that $\int_{M} w_{\infty}^{2}=(4 \pi)^{\frac{n}{2}}$. We shall show that on every compact subset $K$, after passing to subsequences, $w_{i}$ converges to $w_{\infty}$, say in $C^{0}$-fashion. This will imply that $w_{\infty}$ satisfies the equation

$$
-4 \Delta w_{\infty}+S w_{\infty}-n w_{\infty}-2 w_{\infty} \log w_{\infty}=\mu_{\infty}(g) w_{\infty} .
$$

Integration by parts yields that

$$
\int_{M}\left(4\left|\nabla w_{\infty}\right|^{2}+S w_{\infty}^{2}-2 w_{\infty}^{2} \log w_{\infty}-n w_{\infty}^{2}\right)=\mu_{\infty}(g)(4 \pi)^{\frac{n}{2}}
$$

This implies that $\mu_{\infty}(g) \geq \mu(g, 1)$, which is enough to conclude that $\mu_{s} \geq 0$ since $0 \geq$ $\mu_{\infty}(g) \geq \mu(g, 1)=-\mu_{s}$. The claim that $w_{i} \rightarrow w_{\infty}$ in $C^{0}$ norm can be proved using Sobolev embedding theorem (over compact region $K$ ), interior $L^{p}$-estimates, and the compactness of the Sobolev embedding. Since it is rather standard we leave the details to the interested reader. One can also find this in the forthcoming book [Chow, et al2].

Now we point out how one can modify the above argument to the general case. In fact in the proof above the assumption that Ric $\geq 0$ is only used, via Proposition 4.2, to ensure that $\mu(g, \sigma)$ is uniformly bounded for $1-\delta \leq \sigma<1$, for some $\delta>0$. This can be done for the case that $|\operatorname{Ric}| \leq A$ for some $A>0$. We state this as a separate result below. 
Proposition 7.2. Assume that on a complete Riemannian manifold $(M, g), \mu(g, 1)>-\infty$ and Ric $\geq-A$ and $S \leq B$ for some positive numbers $A$ and $B$. Then for any $0<\sigma<1$,

$$
\mu(g, \sigma) \geq \mu(g, 1)-n A \sigma-B-\left(\frac{A^{2} n}{2}+A n\right)(1-\sigma) .
$$

Proof. As in Proposition 4.2, $\mu_{0}(g, 1) \geq \mu(g, 1)-B$. Let $u_{0}(x)=e^{-\tilde{\psi}} /(4 \pi \sigma)^{n / 2}$ be a smooth function with compact support such that $\int_{M} u_{0}=1$. Similarly let $u(x, t)=e^{-\varphi} /(4 \pi \tau)^{n / 2}$ be the solution to the heat equation with $u(x, 0)=u_{0}(x)$. Here $\tau(t)=\sigma+t$. We shall use the entropy formula from $[\mathrm{N} 2]$ to estimate

$$
\mathcal{W}_{0}(0) \doteqdot \int_{M}\left(\sigma|\nabla \tilde{\psi}|^{2}+\tilde{\psi}-n\right) u_{0} .
$$

Let $F(t)=\int_{M}|\nabla \varphi|^{2} u$. The entropy formula of [N2] implies that the entropy

$$
\mathcal{W}_{0}(t) \doteqdot \int_{M}\left(\tau|\nabla \varphi|^{2}+\varphi-n\right) u
$$

satisfies the estimate

$$
\begin{aligned}
\frac{d}{d t} \mathcal{W}_{0}(t) & \leq-2 \tau \int_{M}\left|\nabla_{i} \nabla_{j} \varphi-\frac{1}{2 \tau} g_{i j}\right|^{2} u+2 \tau A F(t) \leq-\frac{2 \tau}{n} \int_{M}\left(\Delta \varphi-\frac{n}{2 \tau}\right)^{2} u+2 \tau A F(t) \\
& \leq-\frac{2 \tau}{n}\left(\int_{M}\left(\Delta \varphi-\frac{n}{2 \tau}\right) u\right)^{2}+2 \tau A F(t)=-\frac{2 \tau}{n}\left(F(t)-\frac{n}{2 \tau}\right)^{2}+2 \tau A F(t) .
\end{aligned}
$$

Viewing the right hand side above as a quadratic polynomial in $X=F(t)-\frac{n}{2 \tau}$, by an elementary consideration we have that

$$
\frac{d}{d t} \mathcal{W}_{0}(t) \leq \frac{A^{2} n}{2}+n A
$$

for $\tau \leq 1$. Hence

$$
\mathcal{W}_{0}(0) \geq \mathcal{W}_{0}(1-\sigma)-\left(\frac{A^{2} n}{2}+A n\right)(1-\sigma)
$$

This shows that

$$
\mu_{0}(g, \sigma) \geq \mu_{0}(g, 1)-\left(\frac{A^{2} n}{2}+A n\right)(1-\sigma) .
$$

Finally we have that $\mu(g, \sigma) \geq \mu(g, 1)-n A \sigma-B-\left(\frac{A^{2} n}{2}+A n\right)(1-\sigma)$.

When $f=$ constant, $(M, g)$ is a compact Einstein manifold with $\operatorname{Ric}_{M}=\frac{1}{2} g_{M}$. The theorem concludes that

$$
\mu_{s}=\frac{n}{2}-\log (V(M))+\frac{n}{2} \log (4 \pi) \geq 0
$$

where $V(M)$ is the volume of $\left(M, g_{M}\right)$. Among all such manifolds the sphere $\mathbb{S}^{n}$ has the smallest $\mu_{s}$. In this case $\mu_{s}$ is monotone non-increasing in $n$ and has the limit $\frac{1}{2} \log \frac{e}{2}$ as $n \rightarrow \infty$, at least for the case that $n$ is even. In fact,

$$
\mu_{s}\left(\mathbb{S}^{2 k}\right)=\log \frac{e^{k}(2 k-1) !}{(2(2 k-1))^{k}(k-1) !} .
$$

It is also easy to see that $\mu_{s}\left(\mathbb{R}^{n}\right)=0$ and $\mu_{s}\left(M_{1} \times M_{2}\right)=\mu_{s}\left(M_{1}\right)+\mu_{s}\left(M_{2}\right)$. 
Remark 7.3. If $(M, g(t))$ is a solution to Ricci flow on compact manifold $M$ over $[0, T)$. Then for any $0 \leq t_{1}<T, \mu\left(g\left(t_{1}\right), T-t_{1}\right) \leq 0$ by an argument similar as (but easier than) the above. For the steady gradient soliton, it is clear that $\lambda \geq 0$ for any steady solitons with $S \geq 0$. We conjecture that if $\mu_{s}=0$, then the shrinker has to be isometric to $\mathbb{R}^{n}$. In [Yo], this conjecture has been proved recently.

Corollary 7.4. Let $(M, g, f)$ be a gradient shrinking soliton as in Theorem 7.1. Then

$$
\int_{M} f \frac{e^{-f}}{(4 \pi)^{n / 2}} \leq \frac{n}{2}
$$

\section{ACKNowledgments}

JAC acknowledges partial support from the project MTM2008-06349-C03-03 from DGIMCINN (Spain) and IPAM (UCLA) where this work was essentially done. LN was supported in part by NSF grant DMS-0805834, Institut Henri Poincaré and an Alfred P. Sloan Fellowship, USA. LN would like to thank Dan Friedan for the lecture [F] on the re-normalization group flow and information regarding the c-theorem. This motivated Theorem 7.1.

\section{REFERENCES}

[AMTU] A. Arnold, P. Markowich, G. Toscani and A. Unterreiter, On convex Sobolev inequalities and the rate of convergence to equilibrium for Fokker-Planck type equations. Comm. PDE. 26(2001), 43-100.

[BE] D. Bakry and M. Emery, Diffusions hypercontractives in Sem. Probab. XIX LNM 1123. Springer, New York, 1985, pp 177-206.

[CH] X.-D. Cao and R. Hamilton, Differential Harnack estimates for time-dependent heat equations with potential. Preprint.

[CHI] H.-D. Cao, R. Hamilton and T. Ilmanen, Gaussian densities and stability for some Ricci solitons. ArXiv: math.DG/0404165.

[CZh] H.-D. Cao, and D. Zhou, On complete gradient shrinking solitons. Preprint.

[CMV] J.A. Carrillo, R.J. McCann and C. Villani, Kinetic equilibration rates for granular media and related equations: entropy dissipation and mass transportation estimates. Rev. Mat. Iberoamericana 19, 3(2003), 971-1018.

[CT] J.A. Carrillo and G. Toscani, Asymptotic $L^{1}$-decay of solutions of the porous medium equation to self-similarity. Indiana Univ. Math. Jour. 49(2000), 113-142.

[ChT] A. Chau and L.-F. Tam, On the simply connectedness of non-negatively curved Kähler manifolds and applications. Preprint.

[CTY] A. Chau, L.-F. Tam and C. Yu, Pseudo-locality for Ricci flow and applications. ArXiv: math.DG/0701153.

[Ch] B. L. Chen, Strong uniqueness of the Ricci flow. ArXiv:0706.3081.

[CLN] B. Chow, P. Lu and L. Ni, Hamilton's Ricci flow. Graduate Studies in Mathematics, 77. American Mathematical Society, Providence, RI; Science Press, New York, 2006. xxxvi+608 pp.

[Chow, et al1] B. Chow, S.-C. Chu, D. Glikenstein, C. Guenther, J. Isenberg, T. Ivey, D. Knopf, P. Lu, F. Luo and L. Ni, The Ricci Flow: Techniques and Applications, Part II: Analytic Aspects. Mathematical Surveys and Monographs, Vol. 144. AMS, Providence, RI, 2008.

[Chow, et al2] B. Chow, S.-C. Chu, D. Glikenstein, C. Guenther, J. Isenberg, T. Ivey, D. Knopf, P. Lu, F. Luo and L. Ni, The Ricci Flow: Techniques and Applications, Part III. Mathematical Surveys and Monographs, to appear, AMS, Providence, RI, 2008.

[CMS] D. Cordero-Erausquin, R.J. McCann and M.A. Schmuckenschlager, Riemannian interpolation inequality 'a la Borell, Brascamp and Lieb. Invent. Math. 146(2001), 219-257.

[DW] A. Dancer and M. Wang, On Ricci solitons of cohomogeneity one. ArXiv: 0802.0759.

[DoPi] J. Dolbeault, M. del Pino, Best constants for Gagliardo-Nirenberg inequalities and application to nonlinear diffusions. J. Math. Pures Appl. 81(2002), 847-875.

[FMZ] F. Fang, J. Man and Z. Zhang, Complete gradient shrinking Ricci solitons have finite topological type. C.R. Aca. Sci. Paris, Ser. I 346(2008), 653-656. 
[FIK] M. Feldman, T. Ilmanen and D. Knopf, Rotationally symmetric shrinking and expanding gradient Kähler-Ricci solitons. J. Differential Geom. 65 (2003), no. 2, 169-209.

[F] D. Friedan, Introduction to the renormalization group flow. Lecture at BIRS, April, 2008.

[Gr] L. Gross, Logrithmic Sobolev inequalities. Amer. J. Math. 97 (1975), 1061-1083.

[H1] R. Hamilton, The formation of singularities in Ricci flow. Surveys in differential geometry, Vol. II (Cambridge, MA, 1993), 7-136, Int. Press, Cambridge, MA, 1995.

[H2] R. Hamilton, Lectures on Ricci flow. Clay Summer School at MSRI, July, 2005.

[LW] P. Li and J. Wang, Weighted Poincaré inequality and rigidity of complete manifolds. Ann. Sci. École Norm. Sup. (4) 39(2006), no. 6, 921-982.

[LV] J. Lott and C. Villani, Ricci curvature for metric-measure spaces via optimal transport. To appear in Ann. of Math. (2) Available online at www.umpa.ens-lyon.fr/ cvillani/.

[Mc] R.J. McCann, A convexity principle for interacting gases. Adv. Math. 128, 1(1997), 153-179.

[Mu] O. Munteanu, The volume growth of complete gradient shrinking Ricci solitons. arXiv: 0904.0798.

[Na] A. Naber, Noncompact shrinking 4-solitons with nonnegative curvature. ArXiv: 0710.5579.

[N1] L. Ni, Ricci flow and nonnegativity of sectional curvature. Math. Res. Lett. 11(2004), no. 5-6, 883-904.

[N2] L. Ni, The entropy formula for linear heat equation. J. Geom. Anal. 14(2004), 87-100; Addenda. J. Geom. Anal. 14(2004), 369-374.

[N3] L. Ni, Ancient solutions to Kähler-Ricci flow. Math. Res. Lett. 12(2005), no. 5-6, 633-653.

[N4] L. Ni, A note on Perelman's LYH inequality. Comm. Anal. Geom. 14(2006), 883-905.

[O] F. Otto, The geometry of dissipative evolution equations: the porous medium equation. Comm. Partial Differential Equations 26(2001), 101-174.

[OV] F. Otto and C. Villani, Generalization of an inequality by Talagrand and links with the logarithmic Sobolev inequality. J. Funct. Anal. 173(2001), 361-400.

[P] G. Perelman, The entropy formula for the Ricci flow and its geometric applications. ArXiv: math.DG/ 0211159 .

[S] K.T. Sturm, Convex functionals of probability measures and nonlinear diffusions on manifolds. J. Math. Pures Appl. (9) 84, 2 (2005), 149-168.

[To] P. Topping, Lectures on Ricci flow. London Math. Soc. Lecture Notes Series 325. Cambridge Univ. Press (2006).

[Vi1] C. Villani, Topics in optimal transportation. Graduate Studies in Mathematics, 58. American Mathematical Society, Providence, RI, 2003. xvi+370 pp.

[Vi2] C. Villani, Optimal transport, old and new. To be published by Springer (2008).

[WW] G. Wei and W. Wylie, Comparison geometry for the Bakry-Emery Ricci tensor. ArXiv: 0706.1120.

[Yo] T. Yokota, Perelman's reduced volume and gap theorem for Ricci flow. Preprint.

[Z] A. B. Zamolodchikov, Irreversibility of the flux of the renormalization group in $2 D$ field theory. JETP Lett. 43(1986), 730 .

\section{ADDRESSES}

José A. CARrillo, ICREA and Departament de Matemàtiques, Universitat Autònoma de Barcelona, E-08193-Bellaterra, Spain

email: carrillo@mat.uab.es

LEI Ni, Department of Mathematics, University of California at San Diego, La Jolla, CA 92093, USA

email: lni@math.ucsd.edu 